\title{
Labyrinthe
}

\section{Le droit est un poème encyclopédique}

L'organisation des savoirs dans la Science Nouvelle de Giambattista Vico

\section{Claudiu Gaiu}

\section{(2) OpenEdition}

Journals

Édition électronique

URL : http://journals.openedition.org/labyrinthe/4059

DOI : $10.4000 /$ labyrinthe.4059

ISSN : 1950-6031

Éditeur

Hermann

Édition imprimée

Date de publication : 1 février 2010

Pagination : 93-104

ISBN : 978-2-7056-6984-3

Référence électronique

Claudiu Gaiu, « Le droit est un poème encyclopédique », Labyrinthe [En ligne], 34 | 2010 (1), mis en ligne le 01 février 2012, consulté le 05 mai 2019. URL : http://journals.openedition.org/labyrinthe/4059 ;

DOI : $10.4000 /$ labyrinthe.4059 


\title{
Le droit est un poème encyclopédique. L'organisation des savoirs dans la Science Nouvelle de Giambattista Vico
}

\author{
Claudiu GAIU \\ clausiro@yahoo.com
}

Le système des connaissances, comme finalité de la rationalité savante théorisée au XVIII ${ }^{\mathrm{e}}$ siècle, contient la tentation constitutive de mettre entre parenthèses l'historicité du savoir. Cet oubli est le point de départ des analyses de Horkheimer et Adorno qui voient dans l'affirmation énergique de la souveraineté de l'individu à l'époque des Lumières la dénégation des origines mythiques de la raison ${ }^{1}$. Par une philosophie qui pense à la fois les notions de système et d'histoire, Giambattista Vico se soustrait à l'accusation des représentants de l'école de Francfort et se recommande comme un héraut de la critique moderne de la dialectique de la raison ${ }^{2}$. Comme les deux penseurs allemands, il voit dans l'accomplissement de la rationalité dans la société les prémisses d'un retour à la barbarie. Néanmoins, il n'explique pas encore cette rechute par l'essence calculatrice de la raison qui soumet aussi bien les choses naturelles que les forces humaines. ${ }^{3}$ Pour le professeur napolitain, le péril vient de l'individualisation égoïste, qui accompagne le progrès du savoir dans toutes les couches sociales. Sa méditation ne s'achève pas non plus sur une phénoménologie de l'aliénation, dont l'heure n'a pas encore sonné, mais dans une généalogie des formes de la rationalité.

\footnotetext{
1. Max Horkheimer, Theodor Adorno, La dialectique de la raison, Paris, Gallimard, trad. Eliane Kaufholz, 1974, p. 16.

2. Le nom de Vico est mentionné en passant à propos de l'origine économique et sociale des concepts de la philosophie grecque (Ibid., p. 38.), mais il y a des correspondances plus patentes entre les deux textes. Ainsi, la mise en évidence de la fonction originairement picturale des mots, dépistée dans les symboles religieux, et l'explication du divorce tardif de la poésie et de la science (Ibid., p. 34-35) se retrouvent dans les théories vichiennes de l'évolution du langage. De même, l'explication de la raison comme radicalisation d'une terreur mythique initiale n'est pas sans rappeler la doctrine de Vico du déploiement de la raison humaine à partir des fables religieuses constituées autour de la panique primordiale provoquée par la foudre jovienne.
}

3. Ibid., p. 94 
La Science Nouvelle $(1744)^{4}$ de Giambattista Vico contient le projet d'une organisation générale des connaissances humaines et d'un dictionnaire des idées produites par l'humanité depuis ses premiers balbutiements. Formées dans le cadre d'une science érudite des lois, et de la passion pour les belles lettres, les idées de Vico vont à l'encontre de la future Encyclopédie française. D'une part, son objet est principalement le monde civil des institutions sociales et politiques. D'autre part, la reconstitution du devenir humain impose des méthodes étrangères à l'esprit des Lumières transalpines: Vico conteste l'utilité de l'explication analytique, incapable de saisir la force générative des concepts et de surprendre le cours de l'histoire. Selon lui, la raison synthétique doit surpasser l'illusion d'un arrêt de l'évolution de la nature, qui pourrait être soumis à une anatomie savante. Par conséquent, ses investigations sur l'ordre des savoirs ne s'accomplissent pas dans une Mappemonde des sciences. La cartographie épistémologique de d'Alembert est ainsi concurrencée par une chronologie de l'esprit humain.

La systématisation des données est assurée par une vision historique et philosophique du droit. La science des lois et des coutumes semble destituer la métaphysique de son rôle organisateur et fondateur des branches du savoir humain. Les difficultés du lecteur contemporain sont liées à l'accouchement difficile des sciences sociales d'une critique radicale du modèle des sciences de la nature. Accusant la partialité des savants qui se sont penchés seulement sur la réalité opaque des choses naturelles, Vico met en premier plan la transparence des institutions créées par l'hommes. À ses yeux, les différents systèmes de la nature et les métaphysiques correspondantes ne sont que des produits récents de l'esprit humain. À la recherche des œuvres originaires de l'humanité, à savoir les formes de la sociabilité, Vico s'empare ainsi du terrain réservé à l'érudition de la science juridique. Les ambiguïtés du texte proviennent de l'occultation de la transformation d'un savoir destiné à soutenir l'autorité par la formation des magistrats et la distribution de la justice dans une histoire critique de la légitimité du pouvoir. Détenant le critère du juste et de l'injuste,

4. Les Principes d'une science nouvelle relative à la nature commune des nations, dont une première version, La Science nouvelle en forme négative (1724), a été perdue, voient le jour 1725. En 1730 Vico retire à son éditeur vénitien une nouvelle version et l'édition définitive ne sort des presses qu'en 1744 , l'année de la mort de l'auteur.

5. Dans le même sens, cf. l'urgence et la nécessité d'un progrès de la science pratique dans Réflexions sur les réformes dans l'administration de la justice (1788) dans le texte de Déborah Cohen, p. 67-68. 


\section{Le droit est un poème encyclopédique}

la science nouvelle nous révèle les rapports de forces dans la société à chaque étape de l'histoire. L'œuvre maîtresse de Vico développe l'idée d'un système du droit naturel des peuples qui organise une théologie civile et rationnelle de la Providence, une philosophie de l'autorité, une histoire des idées humaines, un art critique de la genèse des notions et une science des principes de l'histoire ${ }^{6}$. L'ambition de présenter les principes d'une mise en ordre complète des connaissances humaines, qui anime la Science Nouvelle, est anticipée par des écrits antérieurs qui visaient une réforme de l'enseignement autour d'un «système unique de toutes les disciplines $^{7} \gg$. Plus tard, dans le prologue de son Droit Universel, Vico est toujours à la recherche des fondements d'une « encyclopédie ${ }^{8}$. Si le terme est abandonné, l'idée d'un savoir complet reste constante dans l'évolution de la pensée vichienne.

La question qui nous conduira à travers les dédales de la Science Nouvelle est donc celle de l'ordre des connaissances humaines, décrypté à partir des principes du système juridique universel de Vico. Ce savoir nouveau de l'univers humain organise la synthèse entre la conceptualisation philosophique et la recherche historico-philologique. Il réclame une nouvelle logique capable de rendre compte des articulations de la poésie et du mythe.

Dans son Autobiographie, Giambattista Vico réécrit le processus de la constitution de son système. Premièrement, il éclaircit l'horizon de sa vision. Il admire l'effort théorique sublime des Grecs, qui ont légué à l'humanité les plus fins instruments conceptuels. Mais, pour lui, la métaphysique hellène trouve son couronnement dans la culture historico-juridique latine. La tentative de trouver dans la philosophie première d'Aristote, puis dans celle de Platon, les bases de l'éthique, éveille en lui, « sans s'en rendre compte ${ }^{9} »$, l'intention de concevoir « un droit idéel éternel ${ }^{10} »$, propre à régner sur une cité universelle. La pratique de la métaphysique

6. Le rôle du droit comme «principe architectonique » de la Science Nouvelle est souligné par Jean-Paul Larthomas dans « La "Scienza Nova" comme philosophie du droit », dans Noesis, No 8, 2005, p. 178.

7. De nostri temporis studiorum ratione (1708), xIv, dans Giambattista Vico Opere I, Bari, Laterza, éd. Giovanni Gentile et Fausto Nicolini, 1914, p. 119.

8. De universi iuris. (1720) De opera proloquium, V, 38, dans Giambattista Vico, Opere II-i, Bari, Laterza, éd. Fausto Nicolini, 1936.

9. Autobiografia, in Giambattista Vico Opere V, Bari, Laterza, éd. Benedetto Croce et Fausto Nicolini, 1929, p. 12.

10. Ibid. 


\section{Labyrinthe, $n^{\circ} 34$}

éloigne le penseur de la géométrie et de la logique, disciplines qui consistent dans l'observation attentive des « menues choses ${ }^{11}$ », préoccupation qui fait perdre de vue les catégories de l'être. Contrairement aux esprits analytiques, l'amateur de métaphysique trouvera ainsi satisfaction dans la lecture des orateurs, des historiens et des poètes, qui saisissent la raison commune sans céder à l'abstraction. Par conséquent, si Vico doit au platonisme l'idée d'un droit universel, il cherchera son accomplissement plutôt du côté de la jurisprudence romaine. Deuxièmement, Vico place dans cette perspective les œuvres qui font naître en lui l'idée d'un savoir insolite. Les héros de la formation de l'œuvre vichienne sont Platon et Tacite. Plus tard, ils seront rejoints par Bacon et Grotius. Chacun incarne une idée qui sera développée ultérieurement dans la pensée de Vico. Si Platon est la personnification de la sagesse conceptuelle de la tradition grecque, Tacite représente l'esprit métaphysique qui sait voir dans la réalité les genres philosophiques. Le disciple de Socrate nous expose une morale normative, celle de l'homme tel qu'il devrait être. L'historien latin considère l'homme réel dans son action politique. Vico lui-même précise que l'admiration pour ces deux auteurs était le commencement de la Science Nouvelle comme « histoire idéelle éternelle », schéma du destin particulier de chaque nation ${ }^{12}$. Le chancelier Francis Bacon incarne l'idée encyclopédique d'un savoir contenant toutes les sciences dans le but de constituer une République universelle des Lettres ${ }^{13}$. Mais Vico souligne qu'en dépit de la clairvoyance de l'auteur de Novum Organum, un domaine important avait échappé à son projet: l'ensemble de la vie civile et l'histoire de toutes les nations. Enfin, Vico attribue à Grotius ses propres intentions de transformer la jurisprudence dans une métaphysique civile qui comprend toutes les autres disciplines savantes. Ainsi la science du juste et de l'injuste réunit le savoir conceptuel avec celui des histoires fabuleuses et de la grammaire des langues savantes. Par la méditation sur les principes des lois, le système baconien qui réunissait toutes les disciplines est métamorphosé dans un système du droit universel. Le rôle catalyseur de la science juridique se fonde sur une compréhension élargie du droit, comme étude de l'ensemble des institutions créées par l'homme.

11. Ibid.

12. Ibid., p. 26.

13. Ibid. 


\section{Le droit est un poème encyclopédique}

[...] Hugo Grotius a réuni dans un système du droit universel toute la philosophie et la philologie, toutes les deux parties de cette dernière comprenant autant l'histoire des choses soit fabuleuses soit certaines que l'histoire des trois langues hébraïque, grecque et latine, les seules langues savantes de l'antiquité, qui nous aient été transmises par la religion chrétienne. ${ }^{14}$

La synthèse entre la philosophie et la philologie est donc l'une des prémisses de l'accomplissement du système du droit universel. Cette réunion se produit à partir d'une décomposition critique des deux disciplines, en vue de leur refonte dans une somme des connaissances humaines. Vico reproche aux philosophes de ne pas avoir mis leurs arguments à l'épreuve de la réalité constatée par la philologie, et aux philologues de ne s'être jamais préoccupés d'interpréter leurs constats à la lumière de la raison philosophique ${ }^{15}$. Le philosophe par l'étude des genres est capable de reproduire et de maîtriser par représentation le fait, resté non explicité dans l'observation des philologues. Le privilège des organisations civiles consiste dans leur originalité. Elles ne sont pas simplement la mimêsis de la nature. La nature créatrice de l'homme se manifeste véritablement dans le monde civil - dans les institutions fondées par lui pour mettre en acte sa sociabilité naturelle. La jurisprudence vichienne consiste dans la description de la genèse de l'organisation civile ${ }^{16}$. Elle réclame un art critique nouveau, capable d'investiguer la vérité sur les fondateurs des nations. Cette herméneutique est l'aboutissement de l'accord passé entre le philosophe et le philologue. La philosophie découvre un nouveau domaine, cultivé jusque-là par la philologie, qui réunit l'étude des phénomènes dépendant de la libre volonté de l'homme, comme l'histoire, les langues et les mœurs des nations. L'obscurité des commencements de la vie civile et la diversité des manifestations sociales ont constitué les principaux obstacles à la transformation de la philologie en science. Jusque-là, elle se contentait de la collection des événements et des constatations. Grâce à la systématisation philosophique de ses observations, elle peut maintenant revendiquer une place dans la cité des sciences. Cet ennoblissement académique de la philologie est dû à

\footnotetext{
14. Ibid., 39

15. Science Nouvelle, op. cit., 140.

16. Ibid., 2.
} 


\section{Labyrinthe, $n^{\circ} 34$}

la conversion de la certitude du fait, soutenue par une autorité, en vérité éclairée par la raison. La distinction entre le certain et le vrai reflète la différence entre une position certifiée par une puissance externe (les sens, la coutume, l'autorité politique ou religieuse) et la connaissance dévoilée par l'intellection. La philosophie rend raisonnables les instances non-réfléchies qui gouvernent la vie commune. L'incertitude introduite dans la vie humaine par l'existence du libre-arbitre est compensée par les autorités instituées par le « sens commun ». Cette notion décrit pour Vico un jugement sans réflexion qui caractérise un peuple, une nation ou le genre humain en entier ${ }^{17}$. Ainsi la Science Nouvelle devient l'analyse de la genèse de la rationalité. Elle cherche à travers le témoignage des langues la préhistoire de l'esprit humain. Giambattista Vico inaugure par là une philosophie qui s'intéresse à la vie spirituelle qui précède l'apparition des écrivains et des savants ${ }^{18}$.

La Science Nouvelle opère une division du champ de l'expérience humaine en deux parties: d'un côté la raison, qui produit la science de la vérité, et de l'autre, la volonté, qui engendre la conscience de la certitude $^{19}$, objet des recherches philologiques. Pour Vico, la conscience est l'intériorisation des règles civiques et le siège des expériences sensibles. Elle est en jeu dans l'alliance de la philosophie et de la philologie, qui mène à l'explication rationnelle de l'acceptation des institutions sociales. Expérience psychologique tardive, ultérieure aux représentations sensibles et à l'assimilation des rapports communautaires, la conscience de l'individualité caractérise un âge mur de la réflexion personnelle et de l'histoire humaine. C'est pourquoi la fondation cartésienne de la science dans la certitude de l'existence du sujet pensant ne peut pas servir de point de départ pour une science de la sociétée ${ }^{20}$.

La recherche de la vérité de la science sur la nature commune des nations requiert de nouvelles bases philosophiques et de nouvelles articulations logiques. Ces principes novateurs rendent compte de la rationalité du

\section{Ibid., 142 .}

18. L'orientation du regard vers l'âge mythique des institutions oppose Vico à Voltaire, qui ne voit dans les législations modernes que le résultat hétérogène des influences historiques. Cf. l'article de Déborah Cohen, p. 75.

19. Science Nouvelle, op. cit., 138.

20. Giambattista Vico, De l'antique sagesse de l'Italie (1710), Paris, Flammarion, trad. Jules Michelet, 1993, éd. Bruno Pinchard, I-II, 77-80. Ce titre sera dorénavant abrégé comme suit: De l'antique sagesse. 


\section{Le droit est un poème encyclopédique}

social, qui s'exprime différemment à travers les époques historiques. Leur manifestation éclairée ou obscure marque respectivement l'humanisation ou le retour à la barbarie des communautés. Né au milieu des conventions qui organisent la vie en commun, l'homme est soumis à une éducation qui débute avant l'usage de la parole. L'évolution personnelle, comme l'histoire des nations, est le déploiement rationnel du sens originaire des réglementations civiles. La transformation des institutions sociales des entités imposées par l'autorité communautaire en formules rationnelles, orientées par le bien commun, ouvre l'espace d'une recherche générative. La généalogie philosophique vichienne se fonde sur quelques principes de métaphysique. Dans les Eléments de sa doctrine, Vico précise que la nature des choses ne consiste en rien d'autre que dans l'origine de leur existence, dans des temps particuliers et sous des conditions particulières ${ }^{21}$. Si la temporalité et la manière de l'éclosion ne changent pas, les objets garderont leur identité. Les propriétés inséparables des sujets doivent être produites par les circonstances et les modifications de la naissance des choses. Ces principes de philosophie première ouvrent les portes d'une recherche sur les traditions populaires apparues et maintenues depuis la genèse des nations. Nous reconnaissons derrière la vision dynamique générative de la Science nouvelle un usage remarquable du discours de l'opérationnalité de la métaphysique classique.

Pour contempler les genres et comprendre à partir de ces entités logico-métaphysiques la génération des faits, Vico avait jadis rebroussé chemin vers la sagesse éléate de Zénon ${ }^{22}$. Ainsi, L'antique sagesse de l'Italie se fondait sur les capacités créatrices des concepts. La science produit la vérité à partir de la connaissance des modèles idéaux des choses. L'appréhension par synthèse des concepts nous dévoile toutes les particularités et les accidents qu'ils peuvent recouvrir. Giambattista Vico nous conduit dans une épistémè leibnizienne où la notion d'Alexandre et la notion de Jules César comprennent la vie entière du conquérant de l'Asie et les moindres actions du dictateur de Rome ${ }^{23}$. Nous voyons plus clairement l'une des sources de la conception vichienne des universaux fantastiques. Dans la théorie des mythes, Vico explique les personnages

21. Science Nouvelle, op. cit., 147-148.

22. De l'antique sagesse, op. cit., 92-100. Mais ce Zénon rappelle surtout les théories des entéléchies et des atomes de substances de Leibniz.

23. Leibniz, Discours métaphysique, Paris, Vrin, introduction, texte et commentaire par Georges Le Roy, 1993, art. 8 et 13, respectivement p. $43-44$ et p. 47-49. 
fantastiques par la personnification d'une notion. Dans l'état pré-philosophique de la pensée, l'esprit humain ne peut pas formuler l'idée d'une sagesse dans la vie sociale et civile autrement qu'à travers les légendes sur Mercure Trismégiste ${ }^{24}$. De même dans l'espace de la poésie, la figure de Godefroy, le héros du Tasse, contient la vérité métaphysique de la notion de « guerrier». Les personnages historiques ne peuvent correspondre qu'imparfaitement à l'universel poétique représenté dans l'imaginaire commun par le libérateur de Jérusalem. La poésie fonctionne selon le mécanisme mythologique: elle excite une rationalité ancestrale, qui reste en nous malgré l'éducation philosophique. Sa vérité est d'exprimer l'universalité par le singulier, grâce à sa capacité symbolique. La poésie n'est pas une délectation des esprits cultivés. Elle est l'expression première des langues articulées, où le son des affects était bridé par le rythme du chant et la rime des vers. Il a fallu l'effort libérateur de la philosophie pour permettre à la prose de s'affranchir des cadences des pulsions. Vico transfère les notions complètes d'Alexandre, César ou Homère de l'espace de l'intellect infini et divin dans le monde des traditions culturelles, populaires ou savantes ${ }^{25}$. Vico résout la querelle sur le lieu de naissance de Homère en rendant justice à toutes les cités revendiquant cet honneur. Homère n'a pas une identité physique certaine, mais c'est une « idée poétique ${ }^{26}$ ». Il incarne le sage du monde précivilisé, ses épopées contenant l'histoire mythique de la Grèce ancienne et les sédiments des plusieurs âges de l'humanité hérö̈que ${ }^{27}$.

L'induction et l'analogie ${ }^{28}$, les fondements de l'acquisition des connaissances nouvelles, ne sont possibles que par l'accès préalable à l'universel. Mais l'idée qui permet la généralisation et l'assimilation n'est pas une notion innée, offerte à l'homme au sixième jour de la Genèse. Elle est l'expression poétique des réalités sociales. Le prêtre, le guerrier et le rhapsode sont des figures qui dévoilent les structures de la vie ancienne. Les héros exemplaires qui peuplent les légendes ne sont pas faux si notre attention est orientée vers la vérité idéale exprimée par ces personnages ${ }^{29}$.

24. Science Nouvelle, op. cit., 208.

25. Pour ce qui est du renoncement au rapport à l'intellect divin chez les Encyclopédistes, voir les articles de Marion Chottin, p. 42-43 et d'Arnault Skornicki, p. 57-59, note 27.

26. Science Nouvelle, op. cit., 873 sqq.

27. Ibid., 809.

28. De l'antique sagesse, op. cit., p. 126; Science Nouvelle, op. cit., 122-123.

29. Science Nouvelle, op. cit., 204. 


\section{Le droit est un poème encyclopédique}

Par conséquent, la première branche de la logique vichienne doit être la mythologie ${ }^{30}$. À l'aide de cette discipline, nous pouvons mettre au jour la vérité des fables qui représentent l'histoire des nations païennes et l'origine des sciences. À l'aube de la civilisation, l'humanité n'avait pas d'autres moyens pour narrer son destin à part les mythes. Pour répondre à leurs besoins et à la recherche de l'utile, nos ancêtres ont créé les sciences. Mais le premier langage savant n'est pas distinct de celui des premières histoires fabuleuses. Une telle recherche relève la nature poétique de l'homme ${ }^{31}$. Les transformations impliquées par le procès de rationalisation jettent dans l'oubli le sens originaire des mythes ${ }^{32}$. À la lumière de la critique de Vico, la légende de Daphné traquée par Apollon n'est peut pas être l'histoire d'un vulgaire viol, mais le récit fabuleux de l'institution du mariage, l'un des principaux instruments civilisateur de la réglementation des passions ${ }^{33}$. Pour l'étude de ces modifications des structures psychologiques profondes, le penseur fait appel à l'étymologie. La lenteur de l'évolution des mœurs et des institutions est contenue dans les langues. L'étymologie n'est pas conçue comme une branche de la linguistique. Elle se constitue en tant qu'archéologie des couches de la nature humaine et de ses formes sociales ${ }^{34}$. Dans les mots nous lisons l'histoire de la nature humaine, qui a pris à tour de rôle l'allure de la cruauté, de la sévérité, de la bienveillance, de la délicatesse, et, enfin, de la corruption. Le fondement de la recherche des étapes de la vie est l'observation du développement de l'attention primaire de l'homme pour son propre corps, puis pour les propriétés corporelles et finalement pour l'esprit et pour l'âme.

L'enquête mythologique et étymologique est faite sur le terrain du sens commun, cet assemblage des idées populaires partagées par toutes les communautés. Elle constate un nombre d'opinions identiques entre des peuples qui n'ont entretenu aucun commerce entre eux et en conclut l'existence d'une raison commune des nations. Cette nature universelle de l'humanité est structurée par un «dictionnaire mental », origine de toutes les langues articulées ${ }^{35}$. Le vocabulaire mental décrit l'historicité

\footnotetext{
30. Ibid., 51 ; p. 202.

31. Ibid., 213-214.

32. Ibid., 221.

33. Ibid., 533.

34. Ibid., 22.

35. Ibid., 145, 161.
} 
de la nature humaine. Il commence par les hiéroglyphes symboliques qui ont formé l'écriture corporelle, première forme de communication des peuples en absence d'une langue articulée. Il va jusqu'aux notions philosophiques forgées par la métaphysique. Pour être sûr que les catégories de la philosophie ne sont pas des simples abstractions sans correspondance dans la réalité humaine, la Science Nouvelle examine leur genèse. À première vue, les formules du droit sont une simple application de l'art critique vichien. Le sens commun contient les certitudes du droit naturel, qui constituent la substance universelle des jurisprudences locales. La philosophie est appelée à éclaircir la vérité contenue dans les certitudes de la justice. Parce que la sociabilité est le trait humain essentiel, qui rend compte de la constitution du monde civil, le droit gagne une position privilégiée dans la constitution du projet encyclopédique vichien. L'histoire ne confirme pas l'hypothèse d'une existence solitaire des gens ${ }^{36}$, préalable aux institutions sociales. Cette observation met en difficulté les traditions philosophiques et psychologiques qui ont construit leur notion d'humanité à partir de la considération de l'individu isolé. Engendré directement par la nature commune des nations, le droit naturel reflète une réalité qui a précédé l'abstraction philosophique de l'individualité humaine. Comme tous les aspects de la vie, l'histoire du droit suit les étapes de la rationalisation. La législation naturelle parcourt premièrement l' «âge divin », lorsque la croyance de vivre sous le commandement céleste organise la justice en rapport avec les auspices et les oracles. Elle connaît par la suite l' " âge héroïque », c'est-à-dire des aristocraties dominantes qui oppriment une plèbe considérée naturellement inférieure et essentiellement différente. Enfin, il vient « l'âge du droit » appliqué universellement à une humanité considérée égale par sa participation citoyenne au monde de la culture ${ }^{37}$. La désacralisation du droit signifie le passage de sa certification par des instances extérieures à son acceptation par la lumière intérieure de la raison. Initialement les lois sont respectées en vertu de leur autorité certaine ${ }^{38}$.

Pour mettre en lumière les juridictions des premières sociétés, la Science Nouvelle se transforme dans une " philosophie de l'autorité » qui mène ses enquêtes au-delà de l'apparition des textes écrits ${ }^{39}$. Elle

36. Ibid., 134-135.

37. Ibid., 31 .

38. Ibid., 321-322.

39. Ibid., 350 . 


\section{Le droit est un poème encyclopédique}

reconstitue les formules judiciaires qui ont fonctionné pendant les temps poétiques : les guerres saintes, le duel, le marquage des frontières. L'autorité est initialement assurée par la terreur et la force, exprimée par la religion d'un Jupiter foudroyant ${ }^{40}$. Cette autorité divine est suivie de l'autorité humaine, sous la forme d'un pouvoir de l'homme sur ses pulsions. La notion d'effort (conatus) est retirée du contexte des explications des premiers principes de la nature. Elle y est invoquée pour illustrer la capacité de l'homme de maîtriser et guider les mouvements du corps et de ses passions ${ }^{41}$. Ainsi, l'homme découvre la liberté de sa volonté. Enfin, abandonnant l'errance bestiale, l'homme conçoit une nouvelle forme d'autorité fondée par le droit naturel ébauché autour de la défense des propriétés, surgies de la sédentarisation. Vico reproche aux autres théoriciens du droit naturel - Grotius, Pufendorf, Selden - de ne pas avoir saisi les commencements non écrits de leur doctrine. Peu intéressés par la genèse du droit, ils n'ont pas vu les différences entre les trois âges de l'humanité: la jurisprudence comme interprétation des ordonnances divines par les poètes théologiens ; la législation implacable défendant les intérêts de la caste nobiliaire des héros; et finalement la justice humainement universelle, orientée seulement par le souci de la vérité. Dans le sens vichien, la science du juste et de l'injuste comprend l'ensemble l'histoire humaine.

La Science Nouvelle surpasse par synthèse la division intellectuelle entre l'empirisme philologique et le rationalisme philosophique: la certitude du fait est transfigurée en vérité par sa production à partir du concept. Ce dévoilement des entités rationnelles par l'action, à l'intérieur de l'esprit, par représentation, ou à l'extérieur, dans les institutions civiles, relève du mouvement poétique. Le sens commun contient de manière latente les formes de la sociabilité. L'accomplissement de la raison intellectuelle des nations par la découverte de l'intérêt commun correspond à la révélation de la souveraineté des individus. C'est le premier pas vers la désintégration sociale par l'individualisme. La vision de la lumière rationnelle, comme le midi de la vie des nations, à la fois réalisation du bien commun et commencement de la dissolution des sociétés, constitue

40. Ibid., 387.

41. Ibid., 387, 388, 504 et sqq. 


\section{Labyrinthe, $n^{\circ} 34$}

le caractère tragique de la Science Nouvelle, révélateur de la dialectique de la raison.

La Science Nouvelle contient ainsi le projet d'une encyclopédie des savoirs humains ordonnée de manière chronologique. Sa systématicité se fonde sur un point de vue diachronique. Elle se présente comme une archéologie de l'esprit humain qui découvre dans les couches des traditions les structures successives de l'organisation sociale. La science du droit détient une place de poids dans cette configuration nouvelle grâce à ses potentialités organisatrices. Elle est appelée ainsi parce que la philosophie, qui remplissait traditionnellement le rôle d'organisatrice des savoirs, faisait doublement défaut. Premièrement, elle appartenait à l'histoire de l'esprit humain dévoilée par Vico et ne pouvait pas être elle-même à la fois un effet de ce développement et son principe générateur. Deuxièmement, elle s'est montrée incapable de rendre compte de l'historicité de l'homme. Elle avait interprété les traditions culturelles et l'héritage des civilisations anciennes à la lumière de sa rationalité conceptuelle. Ainsi la philosophie classique avait perdu de vue le sens originaire des institutions humaines. À partir de cette critique de la métaphysique classique, Giambattista Vico opère une synthèse de la philosophie avec la philologie appelée à éclairer les formes et l'évolution de la nature humaine. Le savoir préréflexif des sociétés anciennes s'exprime dans des formes lyriques de la pensée qui précèdent la philosophie. La raison savante est un produit ultérieur des lois, dont la nature est indiquée par le titre d'un chapitre de la Science nouvelle: "Le droit romain a été un poème sérieux et la jurisprudence antique a été une poésie sévère... ${ }^{42} \gg$. La poésie décrit la nature commune des nations et l'origine de la rationalité humaine. Le savoir de Vico est une science totale, qui récupère intellectuellement l'intégralité de l'être humain : les potentialités figuratives du corps, la force symbolique de la parole et l'universalité de la raison.

42. Ibid., 1027. 